University of Nebraska - Lincoln

DigitalCommons@University of Nebraska - Lincoln

February 2004

\title{
Bond Breaking and Temporary Anion States in Uracil and Halouracils: Implications for the DNA Bases
}

Adam M. Scheer

University of Nebraska-Lincoln, ascheer1@gmail.com

Kayvan Aflatooni

Fort Hays State University, kaflatoo@fhsu.edu

Gordon A. Gallup

University of Nebraska-Lincoln, ggallup1@unl.edu

Paul Burrow

University of Nebraska-Lincoln, pburrow1@unl.edu

Follow this and additional works at: https://digitalcommons.unl.edu/physicsburrow

Part of the Physics Commons

Scheer, Adam M.; Aflatooni, Kayvan; Gallup, Gordon A.; and Burrow, Paul, "Bond Breaking and Temporary Anion States in Uracil and Halouracils: Implications for the DNA Bases" (2004). Paul Burrow Publications. 4.

https://digitalcommons.unl.edu/physicsburrow/4

This Article is brought to you for free and open access by the Research Papers in Physics and Astronomy at DigitalCommons@University of Nebraska - Lincoln. It has been accepted for inclusion in Paul Burrow Publications by an authorized administrator of DigitalCommons@University of Nebraska - Lincoln. 


\title{
Bond Breaking and Temporary Anion States in Uracil and Halouracils: Implications for the DNA Bases
}

\author{
A. M. Scheer, K. Aflatooni, ${ }^{*}$ G. A. Gallup, and P. D. Burrow ${ }^{\dagger}$ \\ Department of Physics and Astronomy, University of Nebraska-Lincoln, Lincoln, Nebraska 68588-0111, USA
}

(Received 17 September 2003; published 12 February 2004)

\begin{abstract}
Low energy electrons are capable of breaking bonds in gas phase DNA bases by means of the dissociative electron attachment process. With the aid of new total scattering data in the halouracils and input from quantum chemical calculations, we describe the dipole bound and valence anion states in these compounds and present assignments for the two types of structure appearing in the cross sections. A clear distinction between the two mechanisms for bond breaking is necessary for an understanding of electron induced damage to DNA.
\end{abstract}

DOI: $10.1103 /$ PhysRevLett.92.068102

PACS numbers: $87.50 . \mathrm{Gi}, 34.80 . \mathrm{Ht}$

The ground breaking study of Boudaiffa et al. [1] showed that single and double strand breaks in DNA could be produced through decay of temporary anion states formed by low energy electron attachment. This work, in part, has served to motivate further gas phase studies of electron collisions with the DNA bases, the RNA base uracil, and the halouracils in an effort to understand the fundamental mechanisms involved. Most recently, Hanel et al. [2] (uracil), Denifl et al. [3] (uracil, thymine and cytosine), Abouaf et al. [4] (thymine and 5bromouracil), and Denifl et al. [5] (5-chlorouracil) have examined the electron energy dependence of the production of various anion fragments and made estimates of the magnitudes of the dissociative electron attachment (DEA) cross sections. The radiological significance of the halouracils has been discussed in several of these references and will not be repeated here.

The DEA cross sections of each of the compounds above display sharp peaks, in some cases with large magnitudes, in the $0-4 \mathrm{eV}$ energy range. A few of the features have been attributed above to known $\pi^{*}$ resonances based on their relative energies. Abouaf et al. [4] have ventured a mechanism for the sharpest peaks appearing in the bromouracil and thymine DEA data, suggesting that they are "...vibrational structures due to predissociation of rather long-lived anion states, possibly dipole bound states, by valence $\Pi^{*}$ anion states ...."

In the present Letter, we describe new results in uracil and the halouracils that display structures appearing in the total electron scattering cross sections. We couple these with calculations of the empty valence molecular orbitals as well as properties of the dipole bound states in these compounds to arrive at a comprehensive assignment of the structures. A clear distinction between bond breaking processes that arise through dipole bound anion states and through the $\pi^{*}$ valence anion states is important to make since the former, we argue, is unlikely to play a role in DNA itself.

The temporary anion states associated with occupation of the low lying $\pi^{*}$ valence molecular orbitals of uracil and the DNA bases have been observed previously as structures appearing in the total scattering cross section by Aflatooni et al. [6] using electron transmission spectroscopy (ETS) [7]. However, the connections between these spectra and the structures in DEA mentioned above have not been clearly established. In Fig. 1, we show our

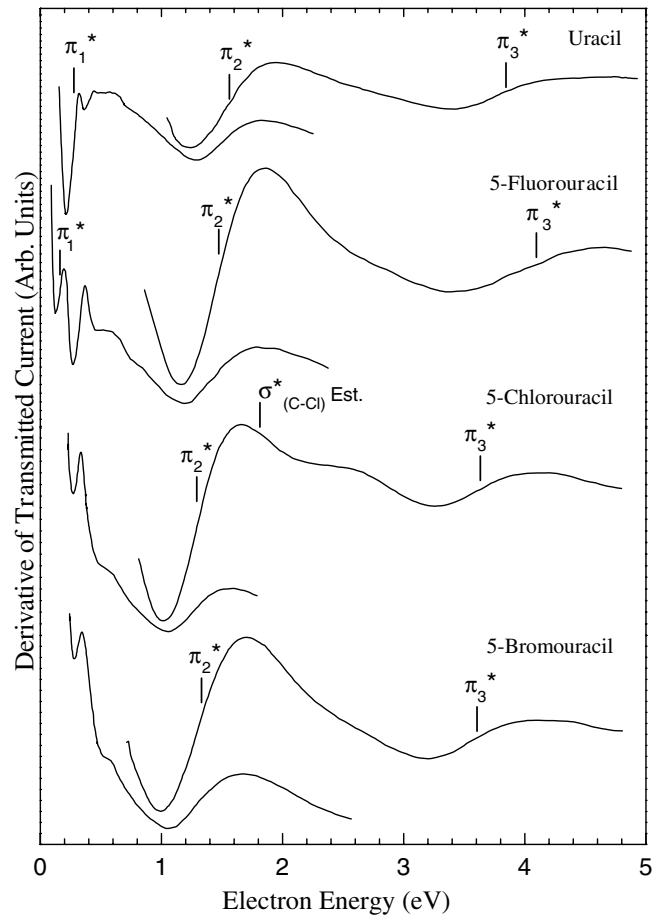

FIG. 1. The derivative with respect to energy of electron current transmitted through uracil, fluorouracil, chlorouracil, and bromouracil as a function of electron energy. Temporary anion states associated with occupation of the $\pi^{*}$ orbitals are indicated. An estimated value for that associated with the $\sigma^{*}(\mathrm{C}-\mathrm{Cl})$ orbital in chlorouracil is shown. Our calculations suggest that the $\pi_{1}^{*}$ anion states of chlorouracil and bromouracil are stable. Pronounced features appear near 0.3 and $0.5 \mathrm{eV}$ in the halouracils that we identify as vibrational Feshbach resonances. 
new ETS data in uracil and the halouracils, plotting the derivative with respect to energy of the electron current transmitted through a cell containing the vapor. The experimental conditions are similar to those used previously [6]. The existence of a peak in the total scattering cross section is signaled in the derivative by a minimum followed at higher energy by a maximum. The midpoint between these extrema is taken as the vertical attachment energy (VAE) for formation of the temporary anion state. The energies of the lowest $\pi^{*}$ anion states are indicated by short vertical lines. As we have found in our previous work [6], 6-31G* basis set constrained calculations of virtual orbital energies (VOE), corrected for continuum interactions [8], provide support for the orbital assignments, and the relative trends in the ETS data for the $\pi^{*}$ orbitals are well reproduced in the scaled virtual orbital energies. The details of these comparisons will be given elsewhere [9]. Our calculations predict that the VAE of the $\pi_{1}^{*}$ anion state of fluorouracil is $0.12 \mathrm{eV}$ and thus we identify the resonance at $0.17 \mathrm{eV}$ with electron occupation of this orbital. The calculations further indicate that the $\pi_{1}^{*}$ anion states of chlorouracil and bromouracil are stable and, hence, not observable in ETS.

Two other aspects of the normally empty valence orbitals are noteworthy. The chlorouracil spectrum displays an additional broad structure just above that for the $\pi_{2}^{*}$ resonance attributable to occupation of the $\mathrm{C}_{5}-\mathrm{Cl} \sigma^{*}$ orbital, the lowest empty valence orbital of $\sigma$ symmetry. (The structure and atom labeling scheme for these molecules is shown in Fig. 2.) The midpoint of this resonance cannot be observed but it can be estimated to lie at approximately $1.8 \mathrm{eV}$ by reference to the position of the

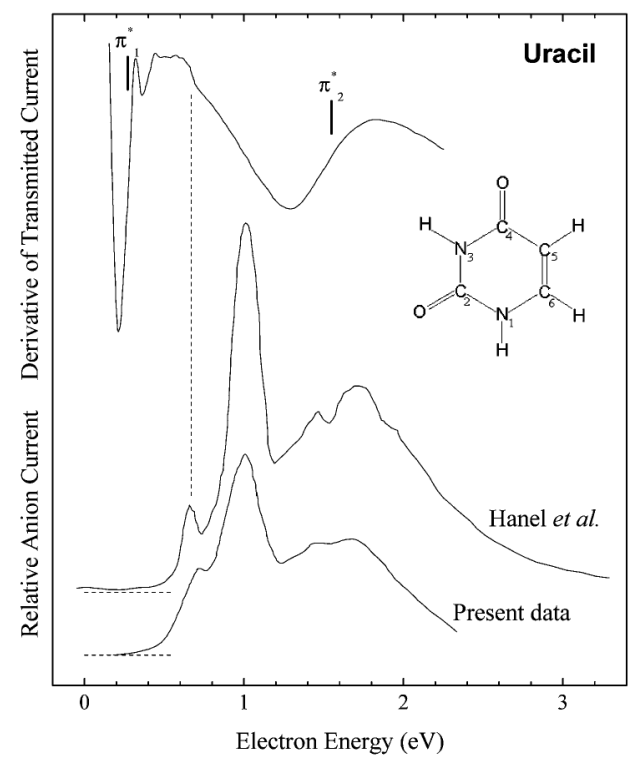

FIG. 2. (upper) Derivative with respect to energy of electron current transmitted through uracil vapor as a function of electron energy. The energies of the two lowest $\pi^{*}$ anion states are indicated. (lower) Relative $(\mathrm{U}-\mathrm{H})^{-}$current as a function of energy. maximum in the derivative signal by means discussed elsewhere [10]. The corresponding $\sigma^{*}\left(\mathrm{C}_{5}-\mathrm{Br}\right)$ orbital in bromouracil is completely obscured by the $\pi_{2}^{*}$ resonance. Of great significance to the present work, the scaled VOE calculations predict the lowest valence $\sigma^{*}$ orbital of uracil to lie near $2.4 \mathrm{eV}$, although the corresponding structure is not observed in the ET spectrum. Such resonances arising from second row elements are generally very short-lived and easily obscured in ETS when $\pi^{*}$ resonances are close by. Plots of the virtual orbital indicate that the wave function of this orbital is largely localized on $\mathrm{C}_{6}$ and $\mathrm{N}_{1}$ and their $\mathrm{H}$ atoms and is antibonding with respect to the ring. Furthermore, the calculated anion energy drops rapidly as the $\mathrm{N}_{1}-\mathrm{H}$ separation increases. Owing to resonance rearrangements in the $\pi$ electron system, the negative charge that might be expected to reside on $\mathrm{N}_{1}$ after the $\mathrm{H}$ atom leaves is distributed widely over the molecule. This occurs more readily for this $\mathrm{H}$ atom than that on $\mathrm{N}_{3}$, and we attribute the low threshold for production of $(\mathrm{U}-\mathrm{H})^{-}+\mathrm{H}$ in uracil, reported by the calculations of Hanel et al. [2] to be $0.8 \pm 0.1 \mathrm{eV}$, to this cause. In fact, the threshold may be as low as $0.6 \mathrm{eV}$, if these authors have ignored the change in zero point energy.

With this brief summary of the empty valence orbitals, we return to Fig. 1 to note the existence of structures near 0.31 and $0.54 \mathrm{eV}$ in the ET spectra of the halouracils. They match quite closely peaks in the DEA cross sections of chlorouracil reported at 0.24 and $0.57 \mathrm{eV}$ [5] and at $0.28 \mathrm{eV}$ in bromouracil [4]. As supported by our VOE calculations, these features cannot be associated with the lowest valence $\pi^{*}$ anion states, and thus we must seek another source. As representative of the DEA process in the DNA bases, we show in the middle part of Fig. 2 the relative DEA cross section of uracil as measured by Hanel et al. [2] and Denifl et al. [3]. These authors showed that at electron energies below $4 \mathrm{eV}$ the uracil cross section is sharply structured and the only anion produced is $(\mathrm{U}-\mathrm{H})^{-}$, the parent molecular anion minus a hydrogen atom. Very similar results are found in thymine. Although our ETS apparatus is not well suited for DEA measurements, we show in the lower part of Fig. 2 the anion current arriving at the walls of the collision cell. The energy resolution is poorer than that of Hanel et al. [2], but the shape confirms their observation. In contrast to the halouracils, very little evidence is seen in the ETS data, shown at the top of Fig. 2, for these narrow structures, although the peak at $0.69 \mathrm{eV}$ in the data of Hanel et al. [2] coincides with a small but sharp decrease in the ETS signal. The vertical dashed line guides the eye for this correlation. Surprisingly, there is no sign of the major DEA feature at $1.01 \mathrm{eV}$, and we return to discuss this later. We note that uracil, thymine, cytosine, and chlorouracil all display peaks in DEA, at $1.01,1.03,1.11$, and $0.99 \mathrm{eV}$, respectively [2-5]. Taken as a whole, the DNA bases and halouracils exhibit sharp features grouped in the ranges $0.24-0.34$, $0.54-0.74$, and $0.99-1.1 \mathrm{eV}$. We show next that these can be associated with vibrational Feshbach resonances 
(VFR), that is, excited vibrational levels of the dipole bound anion states of these compounds.

According to our calculations, the compounds of Fig. 1 possess dipole moments ranging from 4.2 to $4.7 \mathrm{D}$. The dipole bound anion of uracil is stable relative to the neutral by $93 \pm 7 \mathrm{meV}$ as measured by Hendricks et al. [11] and $86 \pm 8 \mathrm{meV}$ by Schiedt et al. [12]. The usual picture of a dipole bound state shows a wave function that is positioned well outside the molecular framework. In the case of uracil, the implication that there is little amplitude on the molecule may be quite misleading. Using a binding energy of $89 \mathrm{meV}$, the radial wave function for a pure dipolar potential field of $4.7 \mathrm{D}$, characteristic of uracil, may be calculated exactly. The corresponding maximum in the function lies at $2.0 \AA$ from the center of mass of the molecule and is inside the $\mathrm{H}$ atoms on the uracil. Clearly, since a pure dipolar potential cannot exist there, that is, in the absence of polarization and other potentials, this implies that the bound state wave function must have considerable valence character. This is consistent with the calculated results of Dolgounitcheva et al. [13] in which a noticeable change in the wave function occurs when the geometry of the anion state is allowed to relax. In spite of the hybrid nature of this state, we will continue to refer to it for convenience as a dipole bound state.

By symmetry, the dipole anion state can mix with states associated with the low lying $\sigma^{*}$ orbitals, and in the case of uracil the lowest of these is the one described above that is calculated to lie near $2.4 \mathrm{eV}$ in the ground state geometry of the neutral. Because this latter anion state is strongly repulsive along the $\mathrm{N}_{1}-\mathrm{H}$ coordinate, the mixing will "drive" the corresponding vibrational mode of the dipole anion state. Furthermore, the mixing is greatly enhanced because of the partially valence nature of the dipole bound state wave function. In the neutral molecule, vibrational motion along the $\mathrm{N}_{1}-\mathrm{H}$ stretch has a characteristic energy of $0.43 \mathrm{eV}$ [14]. Allowing for a measured dipole binding energy of $89 \mathrm{meV}$, we expect the $\nu=1$ level of the dipole bound anion to appear near an electron impact energy of $0.34 \mathrm{eV}$. The structure in DEA cannot appear in uracil at this energy because it lies below the asymptotic energy of separation into $(\mathrm{U}-\mathrm{H})^{-}+$ $\mathrm{H}$. However, because the dipole moments of the halouracils are similar to that of uracil, we expect that these compounds will also possess features near this energy, and as reported here they are consistently observed both in the total and DEA cross sections. Higher lying levels of this mode will be spaced at successively smaller intervals owing to the anharmonicity introduced by coupling between the anion state of the dipole and that associated with the valence $\sigma^{*}\left(\mathrm{~N}_{1}-\mathrm{H}\right)$ orbital. Thus, in uracil we attribute the DEA peak at $0.69 \mathrm{eV}$ to $\nu=2$ and that at $1.01 \mathrm{eV}$ to $\nu=3$. In Fig. 3, we show a schematic of the relevant electronic states of the neutral molecule, dipole bound anion (DBS), valence $\sigma^{*}\left(\mathrm{~N}_{1}-\mathrm{H}\right)$ and $\pi_{2}^{*}$ anions, and VFRs along the $\mathrm{N}_{1}-\mathrm{H}$ stretching coordinate for uracil.

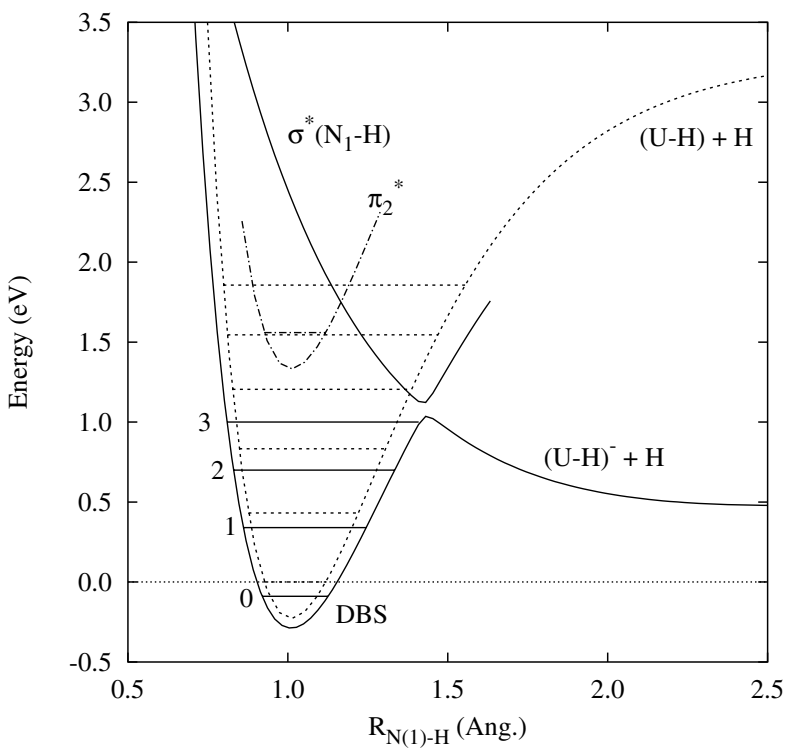

FIG. 3. Schematic potential energy curves for uracil along the $\mathrm{N}_{1}$-H stretching coordinate. The neutral molecule and its vibrational levels are shown as dashed lines, the dipole bound (DBS) and $\sigma^{*}\left(\mathrm{~N}_{1}-\mathrm{H}\right)$ anion states as solid lines, and the $\pi_{2}^{*}$ anion state as a dot-dashed line. At $\mathrm{N}_{1}-\mathrm{H}$ distances greater than $\approx 1.4 \AA$, the fate of the upper resonance curve is uncertain. It may connect with the unobserved $(\mathrm{U}-\mathrm{H})+\mathrm{H}^{-}$asymptote.

In chlorouracil the low lying $\sigma^{*}\left(\mathrm{C}_{5}-\mathrm{Cl}\right)$ anion state observed near $1.8 \mathrm{eV}$ in ETS is strongly repulsive along the $\mathrm{C}_{5}-\mathrm{Cl}$ stretch coordinate. Our calculations show that the wave function of this orbital is also strongly mixed with $\sigma^{*}\left(\mathrm{~N}_{1}-\mathrm{H}\right)$ and coupled to the dipole bound anion state. Because of the lower energy of $\sigma^{*}\left(\mathrm{C}_{5}-\mathrm{Cl}\right)$, the anharmonicity of the VFRs in chlorouracil will be greater than in uracil but may well depend on the anion fragments produced. Thus, $\nu=2$ in chlorouracil is near $0.57 \mathrm{~V}$ in DEA while that in uracil appears near $0.69 \mathrm{eV}$. We expect as well that coupling of the dipole anion with that associated with temporary occupation of $\sigma^{*}\left(\mathrm{C}_{5}-\mathrm{Cl}\right)$ will excite VFRs at the $\mathrm{C}_{5}-\mathrm{Cl}$ stretching frequency. We propose that this accounts for the structure near $0.06 \mathrm{eV}$ in the DEA of chlorouracil [5], but without knowledge of the dipole binding energy we cannot be sure of the vibrational quantum number.

The lifetimes of the VFRs and their visibility in the DEA and total cross sections are easily rationalized in the interpretation presented here. At the $\nu=3$ VFR near $1.01 \mathrm{eV}$ in uracil, the vibrational level will be near the top of the barrier formed by the avoided crossing between the two electronic anion states shown schematically in Fig. 3, and $\mathrm{H}$ atom tunneling through this barrier will be relatively rapid. The resonance in the DEA cross section shows a width of approximately $230 \mathrm{meV}$ [2]. At $\nu=2$, near $0.69 \mathrm{eV}$, the barrier for tunneling is larger and the lifetime of the VFR will be much longer, implying a narrower width. Consistently, the feature in the DEA data of Hanel et al. [2] is narrower, and we argue that its small amplitude may result from its width being less 
than the resolution of the electron beam. As mentioned earlier, our ETS data shows possible evidence for this feature in the total cross section. At $\nu=1$, the VFR is below the threshold for dissociation, precluding its appearance in DEA, and its width is very narrow. Its energy lies so close to the $\pi_{1}^{*}$ resonance that it could be obscured in ETS, even if its lifetime permitted it to be resolved. In the halouracils, this feature is observed in part because the lower lying $\sigma^{*}\left(\mathrm{C}_{5}-X\right)$ valence orbitals couple to the $\sigma^{*}\left(\mathrm{~N}_{1}-\mathrm{H}\right)$ orbital as well as the dipole bound state and thus there are additional decay channels for the VFRs, reducing their lifetimes enough to permit observation of the 0.31 and $0.54 \mathrm{eV}$ features in the total scattering cross section. Decay of the VFRs to vibrational excitation will also play a role, but at present there are no measurements of this channel.

The remaining somewhat broader peaks in the DEA cross sections, for example, the feature underlying the sharp peaks observed in Fig. 2 for uracil, fall close to the VAEs of the $\pi^{*}$ resonances [9]. As observed previously in many studies of DEA in planar halocarbons [15], this process takes place through vibronic mixing of the $\pi^{*}$ states with repulsive valence $\sigma^{*}$ anion states and will not be discussed here further.

Returning now to Fig. 2, the absence of a narrow feature at $1.01 \mathrm{eV}$ in the uracil ET spectrum remains an interesting puzzle. Hanel et al. [2] estimate the cross section of this peak to be $3 \times 10^{-16} \mathrm{~cm}^{2}$, a value that is approximately $25 \%$ of the theoretical maximum for a reaction cross section. Considering that the nearby $\pi_{2}^{*}$ anion state must have comparable size in the total cross section, a peaked structure of this magnitude and width should not be missed in the ET spectrum. It could be argued that Hanel et al. [2] have overestimated the magnitude of this particular decay channel of the resonance. Nevertheless, one would have expected structure in the total scattering corresponding to formation of the resonance. We can suggest only one mechanism for this result, namely, that the resonance structure appearing in the elastic scattering channel has a windowlike profile that cancels the contribution of the DEA channel in the total cross section. There is a precedent for such behavior in electron scattering from $\mathrm{CH}_{3} \mathrm{I}$ [16]. When the computed elastic cross section [17] is added to the DEA cross section presented in Ref. [16], the visibility of the VFR in the total cross section is greatly reduced [18]. This and the many anion state couplings alluded to in the present Letter will provide fertile ground and serious challenges for further theoretical efforts.

In conclusion, we have provided here a specific and consistent description of the structure appearing at low electron energy in recently published studies of DEA in the DNA bases and some of their substituted compounds and in the total scattering cross sections of these compounds. In the unsubstituted bases, it appears that the largest contributions to the measured DEA cross sections in the gas phase arise from the dipole bound states. Given the diffuse form of the dipole wave function, in which much of the charge lies outside the molecular frame, it is very unlikely that these contributions will be preserved when the bases are in DNA. Furthermore, the process yielding such fragments as $(\mathrm{U}-\mathrm{H})^{-}+\mathrm{H}$ does so in part because of facile $\mathrm{H}$ atom tunneling through the barrier. In DNA, the $\mathrm{N}_{1} \mathrm{H}$ does not exist, being replaced by the bond to the sugar ring. In this environment, we expect that the major contribution to bond breaking at low energy, whether local or remote $[19,20]$, will arise from $\sigma^{*}$ predissociation of the valence $\pi^{*}$ anion states.

We are indebted to Ilya Fabrikant for many useful conversations about dipole bound states and VFRs.

*Permanent address: Department of Physics, Fort Hays State University, Hays, KS 67601-4099, USA.

†Electronic address: pburrow1@unl.edu

[1] B. Boudaiffa, P. Cloutier, D. Hunting, M. A. Huels, and L. Sanche, Science 287, 1658 (2000).

[2] G. Hanel, B. Gstir, S. Denifl, P. Scheier, M. Probst, B. Farizon, M. Farizon, E. Illenberger, and T. D. Maerk, Phys. Rev. Lett. 90, 188104 (2003).

[3] S. Denifl, S. Ptasinska, M. Cingel, S. Matejcik, P. Scheier, and T. D. Maerk, Chem. Phys. Lett. 377, 74 (2003).

[4] R. Abouaf, J. Pommier, and H. Dunet, Int. J. Mass Spectrom. 226, 397 (2003).

[5] S. Denifl, S. Matejcik, B. Gstir, G. Hanel, M. Probst, P. Scheier, and T. D. Maerk, J. Chem. Phys. 118, 4107 (2003).

[6] K. Aflatooni, G. A. Gallup, and P. D. Burrow, J. Phys. Chem. A 102, 6205 (1998).

[7] L. Sanche and G. J. Schulz, Phys. Rev. A 5, 1672 (1972).

[8] D. Chen and G. A. Gallup, J. Chem. Phys. 93, 8893 (1990).

[9] A. M. Scheer et al. (to be published).

[10] K. Aflatooni, G. A. Gallup, and P. D. Burrow, J. Phys. Chem. A 104, 7359 (2000).

[11] J. H. Hendricks, S. A. Lyapustina, H. L. de Clercq, J.T. Snodgrass, and K. H. Bowen, J. Chem. Phys. 104, 7788 (1996).

[12] J. Schiedt, R. Weinkauf, D. M. Neumark, and E.W. Schlag, Chem. Phys. 239, 511 (1998).

[13] O. Dolgounitcheva, V. G. Zakrzewski, and J.V. Ortiz, Chem. Phys. Lett. 307, 220 (1999).

[14] P. Colarusso, K.-Q. Zhang. B. Guo, and P. F. Bernath, Chem. Phys. Lett. 269, 39 (1997).

[15] T. Skalicky, C. Chollet, N. Pasquier, and M. Allan, Phys. Chem. Chem. Phys. 4, 3583 (2002), and references therein.

[16] A. Schramm, I. I. Fabrikant, J. M. Weber, E. Leber, M.-W. Ruf. and H. Hotop, J. Phys. B 32, 2153 (1999).

[17] M. Allan and I. I. Fabrikant, J. Phys. B 35, 1025 (2002).

[18] I. I. Fabrikant (private communication).

[19] R. Barrios, P. Skurski, and J. Simons, J. Phys. Chem. B 106, 7991 (2002).

[20] D. M. Pearl et al., J. Phys. Chem. 99, 12379 (1995). 